\title{
IoT Platforms: Analysis for Building Projects
}

Rusu Liviu DUMITRU

Bucharest Politehnica University, Romania

liviu_rusu1964@yahoo.com

This paper presents a general survey of IoT platforms in terms of features for IoT project developers. I will briefly summarize the state of knowledge in terms of technology regarding "Internet of Things" first steps in developing this technology, history, trends, sensors and microcontrollers used. I have evaluated a number of 5 IoT platforms in terms of the features needed to develop a IoT project. I have listed those components that are most appreciated by IoT project developers and the results have been highlighted in a comparative analysis of these platforms from the point of view of IoT project developers and which are strictly necessary as a development environment for an IoT project based. I've also considered the users' views of such platforms in terms of functionality, advantages, disadvantages and dangers presented by this technology.

Keywords: Internet of Things, Cloud Computing, Sensors, Microcontrollers, Wi-Fi Devices

1 Introduction

The Internet of Things (IoT) is the network of physical objects around us that contain electronic components, software, sensors and networking systems, situation that allows these objects to exchange and acquire information. The IoT generally allows the remote control of these objects using the already existing telecommunication infrastructure allowing direct integration into classic computer networks, with immediate benefits at reasonable costs. "Today (2017) it is estimated that there are over 50 billion such objects already connected to the Internet. Through them, it is possible to connect a lot of devices, after interconnecting people through social networks and using the existing infrastructure. It is currently generally used for business, e-learning, education, research, or simply for personal needs" [1].

IoT requires all objects to be equipped with sensors and all people to be equipped with devices to collect this information to send them over the Internet to a series of integrated services to evaluate this information generating a complete map of people's lives. IoT is basically a network of objects interconnected with the following types of relationships:

- People-people

- People - things

- Things - things

What is an IoT platform? It's a suit of components that enables developers to deploy apps, remote data collection, secure connectivity device/sensor MGMT. IoT is a set of sensors, boards, devices that uses network support to exchange data and integrating these ecosystem with cloud platforms lead us to smart objects remotely controlled using collected and analyzed data by these platforms.

\section{Literature Review}

"IoT Platforms (Internet of Things) and Cloud platforms are different technologies but together they are the future of Internet. Merged together and integration enables a large number of applications to be deployed regardless of which operating system is used by the user [2]. Figure 1 describes sensors and devices connected over Internet. Platforms provide a set of features and functionalities that can be used to build IoT, apps facilitating communication between sensors and devices regardless of operating system or type using common working environment storage and applications as virtual solution resides over cloud. Data transmitted between sensors and devices as well as those between devices and cloud-processed-based platforms ensures the business intelligence. "The IoT platform is a hosted service that facilitates deployment of apps without cost of acquiring and complexity of hardware and software layers to ensure interconnecting things based on information and communication technologies by shared networks, apps, storage, hardware and services. 
The survey on IoT platforms was arbitrary based on the most simple and needed features and the options offered to developers."'[3] In the future with the help of IoT, robots will supply information and collaborate with humans by augmented reality and interact more efficiently in taking decisions based on-demand services. IoT can turn every smart object into a useful device that is connected to a network to provide useful information through common communications protocols and friendly interfaces regardless of remotely device used for displaying or control. Today are available various platforms for applications developers used for IoT solutions. The first criterion considered for an IoT platform is that if the platform is an open-source (middleware/platform) or commercial (PAAS) platform. First of all, the developer should consider the following issues:

- Cost of usage (free VS annual fee)

- Visibility, control, interoperability (limited VS free including new features )

- Vendor expertise support (PAAS VS Full stack)

- Deployment (3rd party vs on-premises, cloud, hosted)

- Professional services (expensive, shared VS cost-efficient methodology).

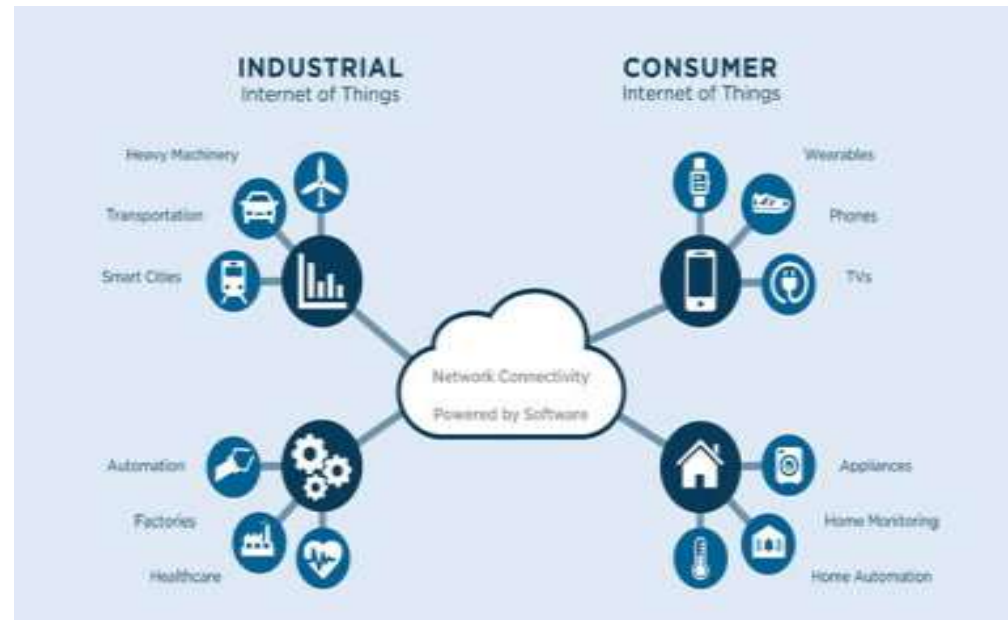

Fig. 1. Connecting sensors and devices trough IoT platforms over Internet [4]

Based on this criterion there are a lot of commercial platforms developed by cloud companies:

- Google Cloud Platforms

- IBM Blue Mix

- Microsoft Azure Cloud

- Amazon Web Services

- Oracle Integrated Cloud

- Bosch IoT suite

- Ericsson Device connection platform

\section{Methods and Analysis}

It is based on an on-line survey of the opensource IoT platforms which are the most successfully used by developers for IoT projects deployments. We used open-source platforms for obvious cost, scalability and control reasons. Of course choosing an IoT platform can be very challenging due to many features and functionalities vendors are offering and a lot of technologies used. Five criterion are important using open-source platforms:

- Technology ownership that is giving to the user full ownership of solution and source code or integration with third-party solution.

- Cost: ensures the free platform with additional support and services free of cost without annual subscription

- New features and full control over the source code of the solution with no lockin proprietary solutions.

- Interoperability and third-party integration: it is addressed to a large community of developers and establish integration with a variety of IoT sensors and hardware devices and can include big-data, mobility solutions and web technologies. 
- Support - cost efficient and dedicated teams and efficient offshore methodology. The on-line survey of the studied platforms envisage 5 main functionalities needed for the development of IoT projects:

- Device management

- Integration

- Security

- Protocols for data collection

- Types of analytics

The five platforms considered in the study are among the most popular open-source platforms used by IoT project developers and 5 major evaluation criteria for IoT platforms have been considered.

The targeted open-source platforms are:

A. KAA IoT platform

B. Particle cloud for Raspberry Pi

C. CARRIOTS IoT platform

D. Everything IoT platform

E. TEMBOO IoT platform

As seen in Table 1 most of the platforms meet most of the necessary criteria:

- Device management

- Integration

- Security

- Data collection protocols

- Types of analytics.

Table 1. IoT platforms comparison based on 5 criteria

\begin{tabular}{|l|l|l|l|l|l|}
\hline $\begin{array}{l}\text { IOT Software } \\
\text { platform }\end{array}$ & $\begin{array}{l}\text { Device } \\
\text { Management }\end{array}$ & Integration & Security & $\begin{array}{l}\text { Protocols } \\
\text { Data } \\
\text { collection }\end{array}$ & $\begin{array}{l}\text { Types } \\
\text { of } \\
\text { analytics }\end{array}$ \\
\hline KAA & No & REST API & $\begin{array}{l}\text { Link encryption } \\
\text { SSL }\end{array}$ & HTTP & $\begin{array}{l}\text { Real-Time } \\
\text { Apache Storm }\end{array}$ \\
\hline Particle & Yes & REST API & $\begin{array}{l}\text { Link Encryption } \\
\text { SSL }\end{array}$ & MQTT,HTTPS & Unknown \\
\hline CARRIOTS & Yes & REST API & $\begin{array}{l}\text { Link Encryption } \\
\text { SSL }\end{array}$ & MQTT,HTTPS & No \\
\hline $\begin{array}{l}\text { Everything } \\
\text { Smart prod- } \\
\text { ucts }\end{array}$ & Yes & REST API & $\begin{array}{l}\text { Link Encryption } \\
\text { SSL }\end{array}$ & $\begin{array}{l}\text { MQTT, COAP, } \\
\text { Web-sockets }\end{array}$ & $\begin{array}{l}\text { Real-Time ana- } \\
\text { lytics } \\
\text { (Rules engine) }\end{array}$ \\
\hline $\begin{array}{l}\text { TEMBOO } \\
\text { Yes }\end{array}$ & REST API & $\begin{array}{l}\text { Link Encryption } \\
\text { SSL }\end{array}$ & MQTT,HTTPS & $\begin{array}{l}\text { Performance } \\
\text { Analytics } \\
\text { (CHOREOS) }\end{array}$ \\
\hline
\end{tabular}

KAA is entirely free of costs, has a capacity to manage millions of sensors, collect and analyze data in real time and visualize it, manage and connect smart products with help of cloud.



Fig. 2. Structure of KAA[6]
It enables data management for connected objects and back-end infrastructure providing the server and endpoint SDK components. KAA provides back-end functionality needed to operate mission-critical IoT solution but has no device management [5].

There are connections made between devices and applications (industrial, manufacturing, services and home apps).Its structure is presented in Figure 2.

Particle is a scalable, reliable and secure IoT platform. A key feature is the ability to run Arduino and Raspberry Pi wiring code making easier to connect electronic components to the cloud but has an unknown type of analytics [7]. 


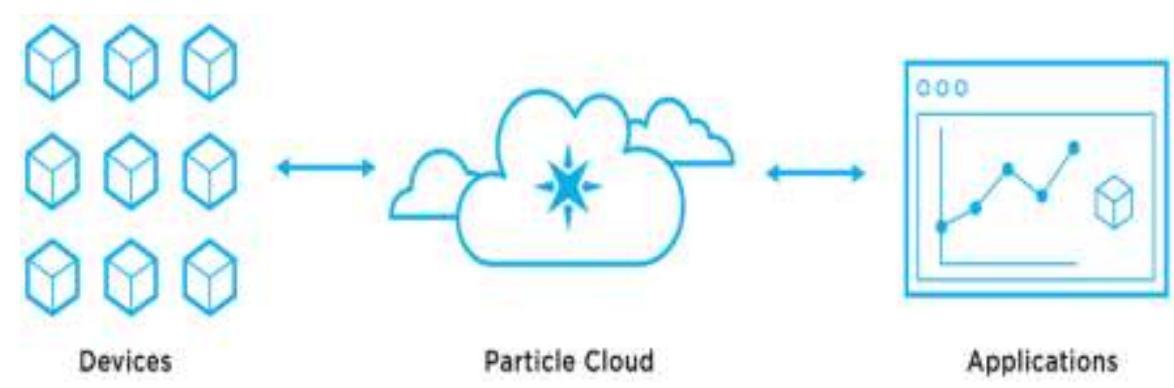

Fig 3 Structure of Particle IoT platform [8]

Its structure, described in Figure 3, shows the devices being brought into contact with the related applications through its cloud platform. CARRIOTS [9]: Easy to connect any device, sending the data to REST API, simple, secured and standard using SDK, Java scripting power at fingertips with Groovy, accessible, integrated and manageable, make it a really powerful platform, remotely maintain control and interact with the devices regardless of their location and check their status, but doesn't have an analytics engine.[10]

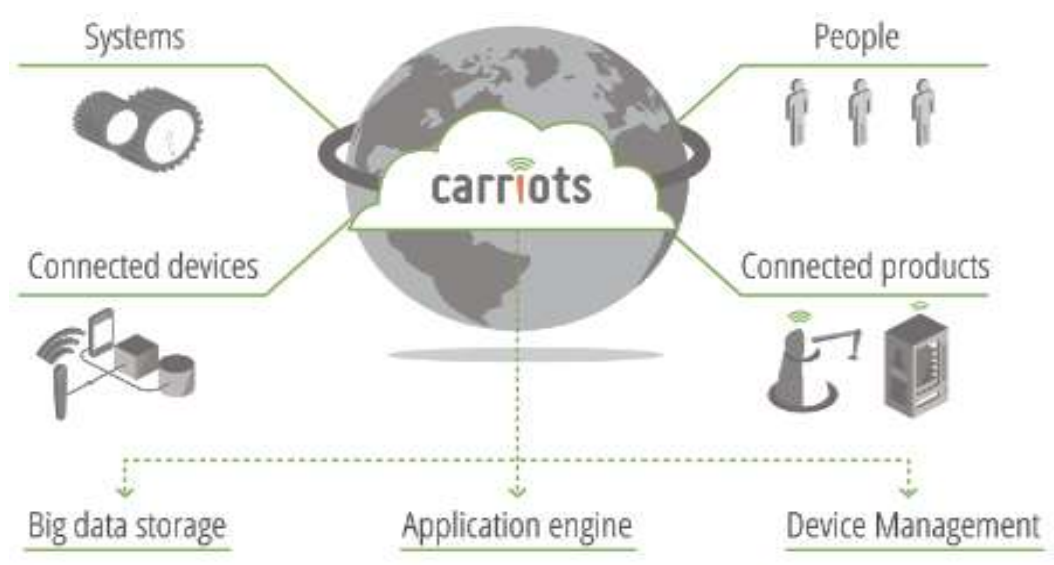

Fig. 4.. Structure of platform [11]

Is picking fast among the developers because it is simply and has easy integration features with external applications. It has Geo-location, dashboard to manage the things, alarm management and SDK development engine. As seen in (Figure 4) Connect devices with users and processing systems through app engine, big data storage and device management.

Everything [12]: It manages millions of intelligent IoT identities given addressable web presence. Reactor is EVERYTHING's IoT brain for smart products and Enterprise Rules Engine makes real-time decision and notifications. It uses dashboard visualization on Business Intelligence tools and KPIs and works with all connectivity technologies (QR, NFC, BLE, RFID) messaging to printed electronics sensor and platform boards. The structure is shown in (Figure 5) 


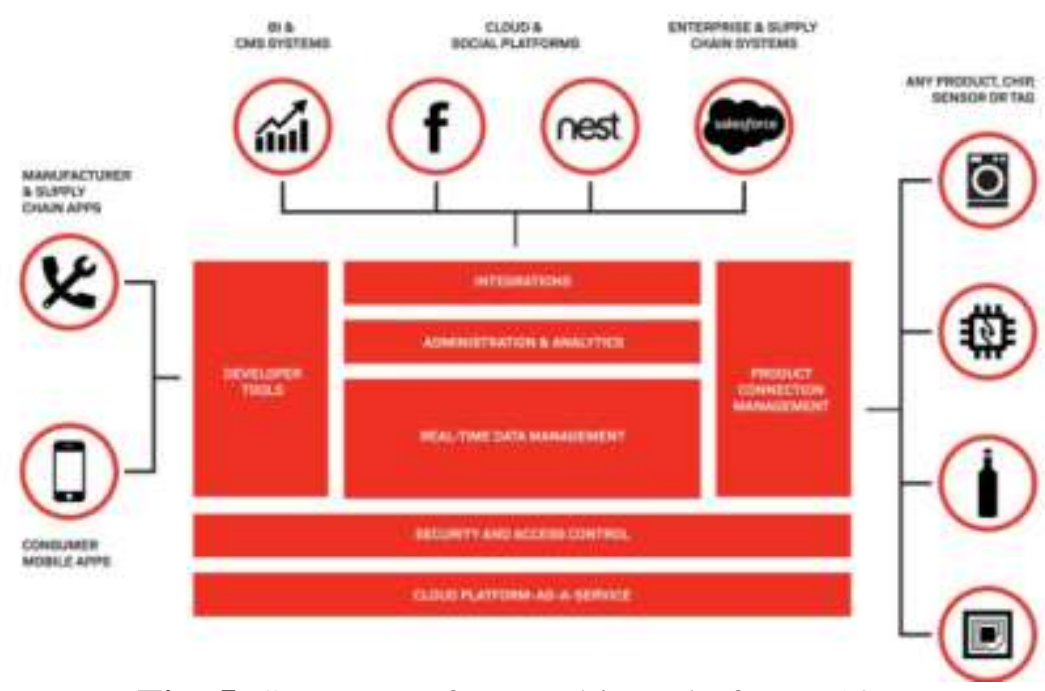

Fig. 5. Structure of Everything Platform [13]

TEMBOO [14]: A mesh network with multiple combinations (MCU's, gateways and devices can be programed and updated with REST API). TEMBOO's structure is shown in Figure 6. It offers lightweight SDKs, libraries and small-footprint agents for programming components (MCU's, SOC's Gateways and mobile apps) for programming in any language on any device, connects $100+$ APIs databases, code utilities all connected through https protocol with the cloud platform.

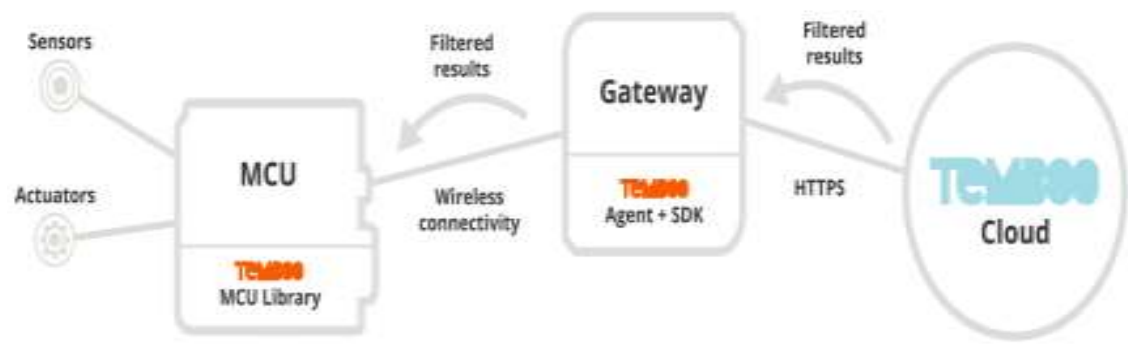

Fig. 6. Structure of TEMBOO platform [15]
Another classification is based on other fea-
- SDK API
tures like (Table 2):
- Data store
- Event /rule management
- Free account
- Data visualization

Table 2 Open source IOT platforms comparison based on another 6 features

\begin{tabular}{|c|c|c|c|c|c|c|}
\hline Platform & $\begin{array}{l}\text { Data } \\
\text { Store }\end{array}$ & $\begin{array}{l}\text { Service } \\
\text { integration }\end{array}$ & $\begin{array}{l}\text { Data visu- } \\
\text { alization }\end{array}$ & $\begin{array}{l}\text { SDK } \\
\text { API }\end{array}$ & $\begin{array}{l}\text { Event/rule } \\
\text { management }\end{array}$ & $\begin{array}{l}\text { Free ac- } \\
\text { count }\end{array}$ \\
\hline $\mathbf{K A A}$ & No & yes & No & Yes & Yes & Yes \\
\hline Particle & Yes & Yes & Yes & Yes & Yes & Yes \\
\hline $\begin{array}{l}\text { CARRI- } \\
\text { OTS }\end{array}$ & Yes & $\begin{array}{l}\text { Yes (email, SMS, } \\
\text { Twitter) }\end{array}$ & No & Yes & Yes & Yes \\
\hline Everything & Yes & Yes & Yes & Yes & Yes & Yes \\
\hline TEMBOO & No & $\begin{array}{l}\text { Yes }(2000 \text { CHO- } \\
\text { REOS) }\end{array}$ & No & Yes & Yes & Yes \\
\hline
\end{tabular}




\section{Result Analysis}

Based on Arduino and Raspberry Pi development platforms users, my research using an online questionnaires were sent to a total of 30 such users asking which of the nominated platforms were used in their projects and a total of 18 users responded to those questionnaires resulting a $60 \%$ accuracy. Based on the 18 respondents I have made a percentage hierarchy of the use of those platforms (Fig. $7)$. KAA ( $15 \%$, because it has no data storage and no data visualization), CARRIOTS (14\%, because it has no data visualization) and TEMBOO (12\%, because it has no data store and no data visualization).

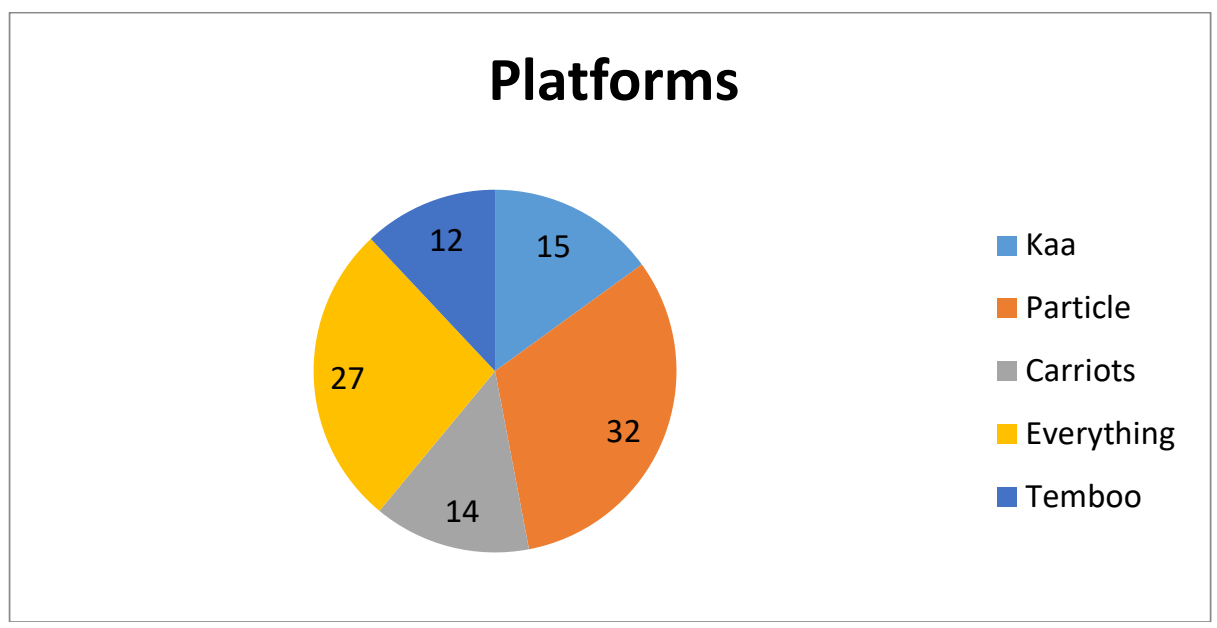

Fig. 7. Popularity hierarchy of 5 open source IoT platforms

In my survey I have included more platforms, the commercial ones too but because my study is based on open-source platforms and due to the fact that most developers were students or passionate only by those platforms I have ranked them according to their popularity. The question was if they heard about those platforms (Figure 8): Do you know any of these platforms?

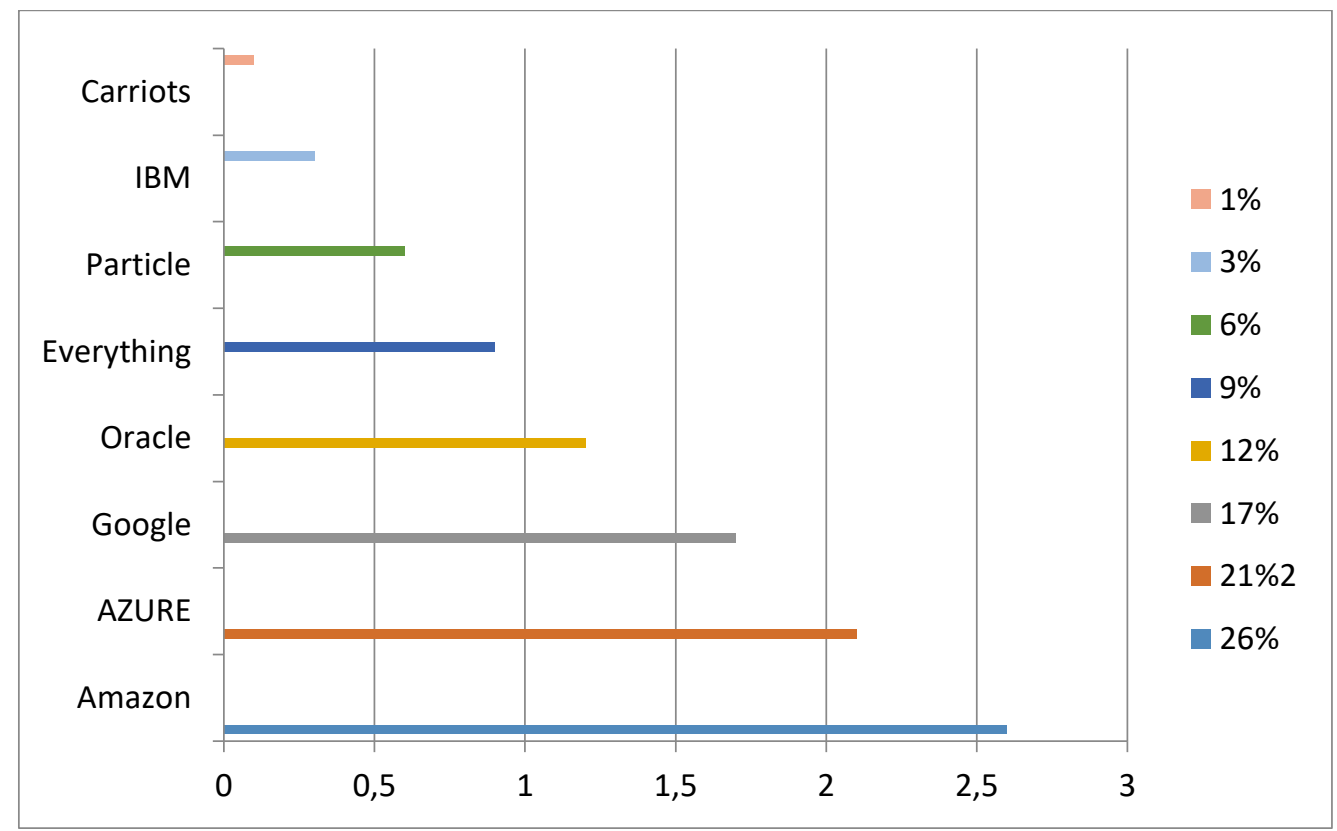

Fig. 8. Popularity Hierarchy of IoT platforms both open-source and commercial

Based on their opinions (fig 8), most of them know better the commercial development 
platforms, but because of the subscription to be paid, many prefer only open-source variants, just those who develop professional applications use commercial variants. However, with the decline in cloud services and IoT services prices, the popularity of commercial development platforms increases together with the acquisition of open-source variants as a move to increase market share. Regarding the popularity of open source platforms, respondents indicated the hierarchy based on the ease of connection and protocols recognized by the Arduino and Raspberry Pi platforms (widely used by respondents) and this explains the hierarchy described in Figure 9.

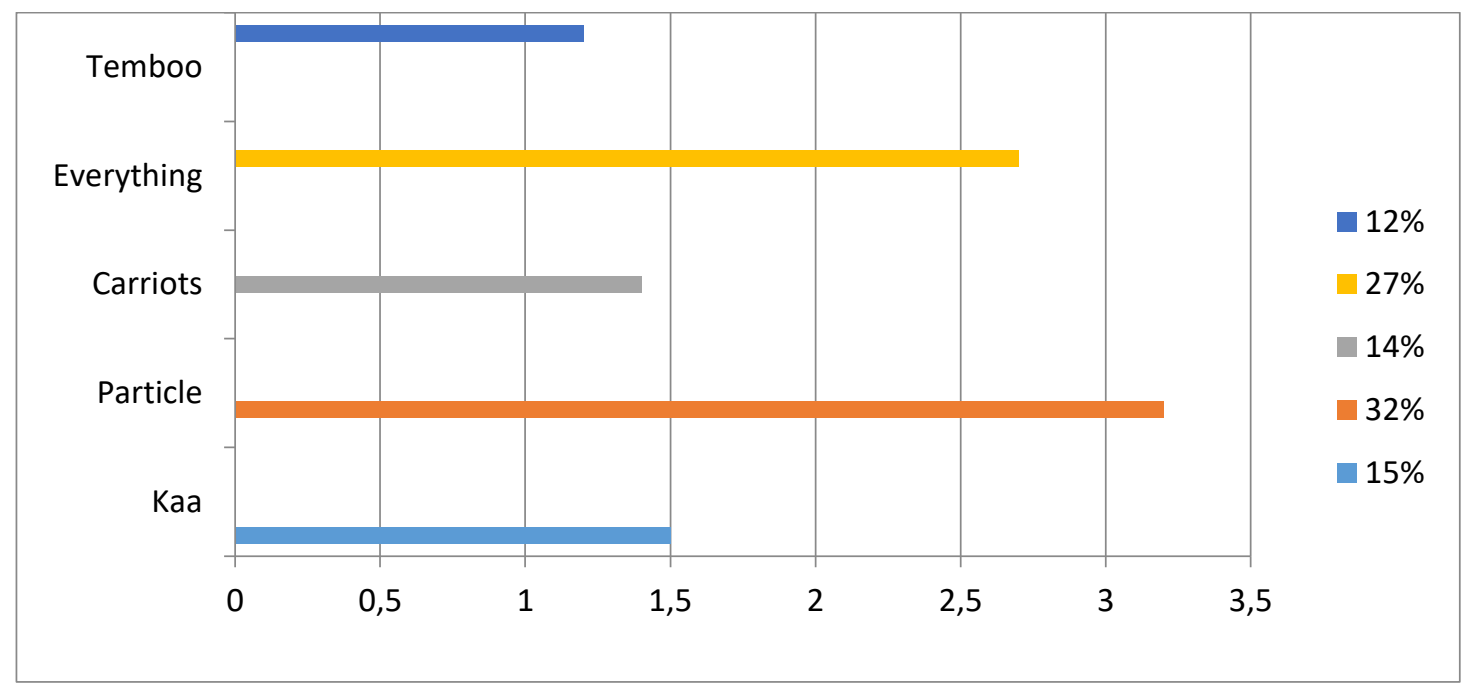

Fig. 9. Widely used open-source IoT platform hierarchy

Based on ease of connection and protocols recognized by ARDUINO and Raspberry Pi development platforms. Of course a platform needs more features to be popular among development users not only those described in this article. Other feature are described below (Fig 10).

- Infrastructure

(IPV4,IPV6,6LowPAN,RP)

- Identification (EPC,uCode,Ipv6,URIs)

- Communication/Transport (Wi-Fi, Bluetooth, LPWAN)

- Discovery (Physical Web, MDNS, DNS$\mathrm{SD})$

- Data Protocols (MQTT, COAP, websocket, HTTP, Https)

- Device management TR-069,OMA-DM)

- Semantic (JSON-LD,WEB Thing Model)

- Multi-Layer Frameworks (ALLJOYN, IOTIVITY, Weave, HOMEKIT)

- Security (Connected Home, Industrial)

Feature to improve on:

It's clear that are many features in which improvement are needed. Some of them are be- ing implemented already by IoT vendors, others are in progress. One of the most important features is the user interface and I'm not thinking just about wearable like smart watches, bracelets, drones, VR glasses, or smart Health Products that all uses screens.

Thinking beyond screen is the new trend

- Knock anywhere is an interface via KNOCKI as a small wireless device that triggers based on how many times a user knocks on the surface.

- Button as interface POP Home Switch (Logitech), serve as a capable home automation hub for bulbs, door locks speaker (as disparate devices) that are designed to unite devices together.

- Hands as an interface via AUGUMENTA: That takes a different approach combining hardware (connected eyewear glasses), software and augmented reality and user's body as an interface. Wen glasses are used, hands of user become configurable dashboard, keyboard, sliders etc. to operate machine controls.

- In-ear digital assistances interface, via 
Sony's XPERIA as a Sony wearable the EARBUDS are wireless assisting navigation, music search, weather, scheduling devices equipped with accelerometers and gyroscopes.

- Talking Pill Bottle as an interface (Adhere Tech) a connected pill bottle preconfigured by the pharmacist provided with audio and visual reminders to take the medications and medical alerts when patients fail to do so. [16]

\section{Discussion}

For the user it is very important that the interface to be friendly and accessible regardless of the device on which it is deployed and regardless of the operating system used by the device.

“Integration is all about API's: this allows applications to communicate with the devices regardless of the type or manufacturer. API's data allow the device to transmit data acting as a data interface and to control the device as a function interface. APIs can be used to sync data locations at levels in the hierarchy like:

- A single infrastructure that include all the devices

- A unique device in the structure

- Group of the data values

- A single data value

What we want to achieve?

To develop UIs for IoT based projects for the next generation of devices. How should this look like?

- One app to rule them all

- Design for occasional use

- Design links between devices

- Increasing opportunities through different IoT user interfaces designs like:

"Screen-less connected devices to improve analog interactions, implemented gesture and movements to streamline interactions and facilitate multiple users to data-driven business models." [17] Almost the same importance is Internet connection because all these sensors and devices need to communicate: IoT communication protocols [18].

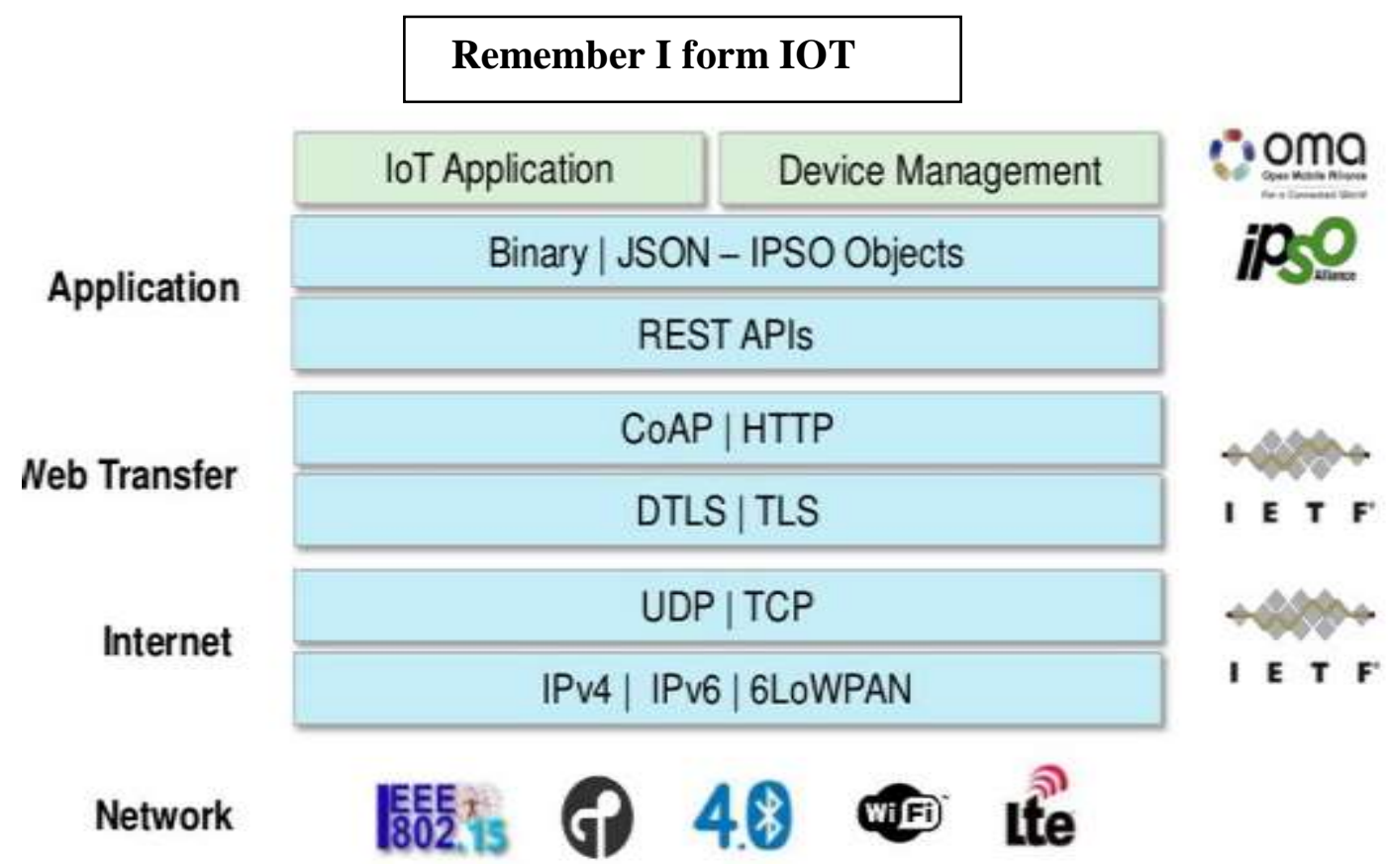

Fig. 10. IoT protocols [19]

Problems of existing IOT cloud platforms: There are a lot of challenges for existing platforms:

- Standardization (lack of it)

- Heterogeneity network platforms
- Context awareness lot of sensors and data

- IoT node identity

There are still a lot of feature to improve:

- Data analytics (a lot of IoT platforms support real time but interactive data may be 
just as important

- Benchmark ( to evaluate and characterize the system performance)

- Edge analytics (to improve communication and reduce the network bandwidth for data transmitted between sensors and devices or IoT server.

- Other issues (legal concern about stored personal data and security)

or to add:

- Handling out of order processing

Errors or malfunction of sensors or network delays, network latency, clock drift and the event processing results in system failure

\section{- Support for IoT context}

Is based on past behavior, stated preferences, location for an individual that has access to rich context information from various sensors and IoT analytics should take it into consideration [20].

\section{Conclusions}

From this article we can conclude that most of the commercial platforms are very well known by project developers, Amazon and Microsoft Azure are the most promising and Google most popular for the Google app engine and IBM despite it has little visibility has a powerful management support and app creation (fig 8 ). The popularity of commercial development platforms will grow in the future by improving service delivery and lowering prices.

For the open-source IoT platforms the tables (1 and 2) above summarize some aspects of these platforms that IoT project developers think that are important. The aim of the comparison provides some technical information or popularity about existing IoT platforms and users should read carefully each platform directly on the site before any use of it (Figure 9). Meanwhile, many other development platforms, both commercial and open-source, have emerged that have not been included in this research and can have features or facilities appreciated by developers. There are several IoT platforms with different services but each platform has unique aspects and it's up to project developers to choose the right one accord- ing to their project. For developers it is necessary to update their knowledge about the IoT platforms before using them based on other features besides those focused aspects in this research ( device management, integration, security, protocols for data collection or types of analytics) first comparison or (data store, service integration, data visualization, API SDK, event management, free account ) second comparison. Another problem rise from use of IoT is security vulnerabilities. Authentication protocol is one of this aspect but each connection protocol has its own weaknesses. Solving the security problems of IoT platforms it is a challenge for developers.

\section{References}

[1] P. Roberts and P. Srinivasan "Log Me In, A Guide To Designing Resilient Products for Internet of Things" published in Technology on Feb 24, 2015

[2] V. Gazis , M. Gortz, M. Huber, K. Mathiudakis, A. Wiesmaier, F. Zeiger and E. Vasilomanolakis "A survey of technologies for the Internet of Things", in Proc. Wireless communication and mobile computing conference (IWCMC 2015 Dubrovnik Croatia)

[3] P.P. Ray (2017 Feb 01) “A survey of IOT cloud platforms". Future Computing and Informatics Journal 06.03. 2017 available online: https://doi.org/10.1016/j.fcij.2017.02.001

[4] What is the Internet of Things (IoT)? IoT definitions and terminology, available at: https://www.i-scoop.eu/internet-ofthings-guide/internet-of-things-definition/:

[5] Kaa IoT Development Platform overview Cyber-Vision, Inc.10295 Collins Avenue, Suite 804, Bal Harbour, FL 33154 available at: https://www.kaaproject.org/overview/30-04-2107

[6] Kaa Platform Structure available at: https://www.kaaproject.org/overview

[7] Particle IoT Platform San Francisco Business available: https://www.particle.io/

[8] Particle IoT platform structure available at: (https://www.particle.io/) 
[9] CARRIOTS platform as product of CARRIOTS S.L. Spain a division of WAIRBUT available at: https://www.carriots.com/

[10] CARRIOTS technical presentation Building an internet of things Project available at: https://www.carriots.com/newFrontend/img-carriots/press_room/ CARRIOTS _technical_ presentation.pdf

[11] CARRIOTS platform structure available at: $\quad$ https://www.carriots.com/newFrontend/img-carriots/press_room/carriots_technical_presentation.pdf

[12] Everything Solutions Platform available at: https://evrythng.com/platform/features/

[13] Everything Platform Structure available at: https://evrything.com/platform/features/

[14] TEMBOO Platform 104 Franklin Str. New York 10013 at: https://temboo.com/platform

[15] TEMBOO Platform structure available at : https://temboo.com/platform

[16] J. Groopman "The IOT user interface designs: Thinking beyond the screen" in
Techtargets IoT Agenda, a topic platform available at: http://internetofthingsagenda. techtarget.com/feature/ The-IoT-user-interface-designs-Thinking-beyond-thescreen

[17] D. Linthicum "App nirvana: When the Internet meets the API economy in TechBeacon topic platform available at: https://techbeacon.com/app-nirvanawhen-internet-things-meets-api-economy

[18] S. Ford - Director of IOT Platforms ARM "Architectures/Graphic" in Postscapes topic platform Internet of Things protocols available at: https://www.postscapes.com/internet-of-things-protocols/

[19] S. Ford - Director of IoT Platforms " $A r$ chitectures/Graphic IoT protocols" in Postscapes topic platform Internet of Things protocols available at: https://www.postscapes.com/internet-ofthings-protocols/

[20] M. Dayarathna "Comparing 11 IOT Development platform" published in DZone/IoT Zone topic platform in Feb.04.2016 available at: https://dzone.com/articles/iot-softwareplatform-comparison

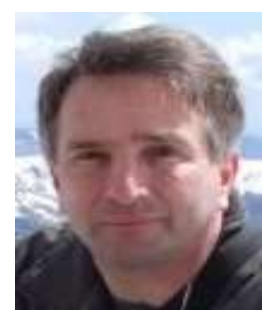

Rusu Liviu Dumitru has graduated the Master Program of Economic Informatics at Faculty of Economic Cybernetics, Statistics and Informatics at Bucharest University of Economic Studies in 2016 and the Master Program of Databases and Web Technologies at Faculty of Mathematics from Bucharest University also in 2016 and currently he is student in the second year at the Master program of Internet Engineering Systems Department of Computer Science at Politehnica University Bucharest. In 2016 he followed an Internship as web developer at Infrasoft Company using Java and Java script for an electronic document archiving platform. 